\title{
Recubrimiento comestible a base de alginato en combinación con eugenol nanoencapsulado y su efecto conservador en la vida útil de jitomate (Solanum lycopersicum)
}

\author{
Alginate-based edible coating in combination with nanoencapsulated eugenol and its preservative \\ effect on the shelf life of tomato (Solanum lycopersicum)
}

\author{
Andrés Mauricio Piña-Barrera', Marbella Saraí Ramírez Pérez², Rocío Álvarez Román ${ }^{3}$, Juan Gabriel Báez González², \\ Carlos Abel Amaya Guerra², Sergio Arturo Galindo Rodríguez* \\ Facultad de Ciencias de la Salud. Universidad de Montemorelos, Montemorelos, 67515, Nuevo León, México. \\ 2 Facultad de Ciencias Biológicas. Universidad Autónoma de Nuevo León, San Nicolás de los Garza, 66450, Nuevo León, \\ México. \\ 3 Facultad de Medicina. Universidad Autónoma de Nuevo León, Monterrey, 64460, Nuevo León, México.
}

\section{RESUMEN}

Las deficiencias en la tecnología postcosecha y el ataque de fitopatógenos ocasionan que los productos hortofrutícolas, como el jitomate, presenten una vida útil muy corta. Además del daño económico, esto puede traer efectos negativos sobre la salud y el medio ambiente. El objetivo de este trabajo fue evaluar un recubrimiento activo de alginato de sodio en combinación con nanocápsulas poliméricas cargadas con eugenol (AL-NP-EUG) para mejorar la vida útil del jitomate. Utilizando la técnica de nanoprecipitación se obtuvieron NP con un tamaño de $171 \mathrm{~nm}$, un índice de polidispersidad de 0.113 y un potencial zeta de $-2.47 \mathrm{mV}$. Utilizando la técnica de HS-SPME con CG-FID, se determinó un porcentaje de eficiencia de encapsulación de $31.85 \%$ para el EUG. El estudio de vida útil demostró que los jitomates tratados con el AL-NP-EUG mantuvieron por más tiempo la firmeza que aquellos sin el recubrimiento. Además, en el ensayo de patogenicidad se demostró que los jitomates con el AL-NP-EUG no presentaron signos de daño ocasionado por el fitopatógeno Colletotrichum gloesporoides. Se concluyó que la formulación de EUG nanoencapsulado e incorporado al recubrimiento comestible presenta alto potencial para su aplicación como nanoconservador natural de productos hortofrutícolas como el jitomate.

Palabras clave: Vida útil, recubrimientos comestibles, eugenol, nanopartículas poliméricas y HS-SPME.

\section{ABSTRACT}

Deficiencies in post-harvest technology and the attack of phytopathogens cause a very short shelf life of fruits and vegetables, such as tomatoes. In addition to the economic damage, it can have negative effects on health and the environment. The objective of this work was to apply an active sodium alginate coating in combination with eugenolloaded polymeric nanocapsules (AL-NP-EUG) to improve the shelf life of tomato. Using the nanoprecipitation technique, NP were obtained with a mean size of $171 \mathrm{~nm}$, a polydispersity index of 0.113 and a zeta potential of $-2.47 \mathrm{mV}$. Using

*Autor para correspondencia: Sergio Arturo Galindo Rodríguez Correo electrónico: sagrod@yahoo.com.mx

Recibido: 6 de abril de 2021

Aceptado: 18 de agosto de 2021 the technique of HS-SPME coupled to GC-FID, a percentage of encapsulation efficiency of $31.85 \%$ was determined for the EUG-loaded NP. The shelf life study showed that tomatoes treated with AL-NP-EUG held firm longer than those without the coating. In addition, for the pathogenicity test, it was shown that tomatoes with AL-NP-EUG did not show signs of damage caused by the phytopathogen Colletotrichum gloesporoides. It was concluded that the formulation of EUG nanoencapsulated and incorporated into the edible coating has high potential for its application as a natural nanopreservative of fruit and vegetable products such as tomato.

Keyword: Shelf-life, edible coatings, eugenol, polymeric nanoparticles, and gas chromatography.

\section{INTRODUCCIÓN}

México actualmente se encuentra entre los 10 principales países productores de productos agroalimentarios a nivel mundial. El jitomate (Solanum lycopersicum) es uno de los productos hortofrutícolas más importantes de México. Lamentablemente, su misma naturaleza, las deficiencias en la tecnología postcosecha y el ataque de fitopatógenos ocasionan que, en general, los productos hortofrutícolas, presenten una vida útil muy corta y pérdidas postcosecha de hasta el 50\% de la producción total, según información de la Organización de las Naciones Unidas para la Agricultura y la Alimentación (FAO, 2011). En los últimos años, el uso de conservadores naturales en la industria alimentaria se ha vuelto tendencia debido a la demanda de los consumidores por los productos naturales. En particular, los aceites esenciales (AE) son metabolitos secundarios de las plantas aromáticas y tienen una amplia gama de actividades biológicas (e.g. antioxidante y antimicrobiana) que los han hecho emerger como una alternativa para el control y reducción de pérdidas postcosecha (Pandey et al., 2017; Ju et al., 2019). De hecho, las propiedades antimicrobianas de los $\mathrm{AE}$ y sus componentes se han aprovechado para controlar hongos y bacterias fitopatógenas (Camele et al., 2012; Choudhary et al., 2017). El eugenol (EUG) es un derivado fenólico conocido comúnmente 
como esencia de clavo, que se extrae de los AE de pimienta, laurel, canela y alcanfor, entre otros. Se ha demostrado que el EUG presenta una actividad antimicrobiana importante contra bacterias y hongos (Marchese et al., 2017), además, la Administración de Alimentos y Medicamentos de los Estados Unidos (FDA) lo ha clasificado como sustancia GRAS (Generalmente Reconocido Como Seguro) y ha sido aprobado por la Comisión Europea como aditivo en alimentos (Prates et al., 2019). Lamentablemente, la aplicación de compuestos como el EUG, como aditivos alimentarios, tiene limitaciones ya que presentan un fuerte carácter lipofílico, alta volatilidad, son insolubles en agua y se deterioran con facilidad por factores ambientales, como la luz y el oxígeno, lo que dificulta su incorporación en productos comerciales (Asbahani et al., 2015; Feyzioglu and Tornuk, 2016). En años recientes, la nanotecnología en la industria de los alimentos ha presentado un desarrollo importante, ofreciendo nuevas alternativas para superar estos impedimentos. La nanoencapsulación supone la incorporación, adsorción, solubilización o dispersión de compuestos bioactivos (e.g. EUG) en o sobre una estructura polimérica a escala nanométrica. La incorporación de estos biocompuestos en nanopartículas poliméricas (NP) a base de polímeros preformados (e.g. Eudragit L 100-55) puede protegerlos contra la degradación, mejorando así su estabilidad física y química. Además, la combinación de estas NP con los denominados recubrimientos comestibles (RC) ha surgido como una alternativa importante en la conservación de alimentos. Un RC se define como una capa continua y delgada, de algún material, que es incorporada sobre el alimento. El alginato (AL) es un polisacárido aniónico lineal glicosídico constituido por unidades monoméricas de $D$-manuronato y L-guluronato y es obtenido principalmente de dos fuentes: las algas pardas (Phaeophyceae) (40\% de la materia seca) y bacterias (Guerreiro et al., 2015; Zia et al., 2015). Este polímero se ha utilizado en la industria alimentaria como material de recubrimiento o empaque, además, también es reconocido por la FDA como sustancia GRAS. Se ha estudiado la incorporación de nanomateriales y antimicrobianos, incluidos aceites esenciales y sus componentes, en RC para dar nuevas propiedades al recubrimiento y mejorar la seguridad y vida útil de frutas y verduras (Correa-Pacheco et al., 2017; PiñaBarrera et al., 2019). En este contexto, un sistema combinado de NP, RC y compuestos que exhiben actividad biológica, como el EUG, puede tener potencial como alternativa a los conservadores agroquímicos sintéticos.

Por lo anterior, el presente estudio se enfocó a evaluar la eficacia de un sistema combinado de NP-EUG con un RC de alginato para extender la vida útil del jitomate (Solanum lycopersicum) e inhibir la acción fitopatógena de Colletotrichum gloesporoides.

\section{MATERIALES Y MÉTODOS Materiales}

El alginato (PM 216,12 $\mathrm{g} \mathrm{mol}^{-1}$ ) y el EUG (Eugenol Reagent Plus $99 \%$ ) se adquirieron de la marca Sigma Aldrich ${ }^{\circ}$. El alcohol polivinílico (Mowiol 4-88 con un PM 26,000 $\mathrm{g} \mathrm{mol}^{-1} \mathrm{y}$ grado de hidrolisis de $88 \%$ ) fue amablemente donado por Omya AG. Se utilizaron jitomates (Solanum lycopersicum) comerciales los cuales presentaron características homogéneas de color, textura y tamaño para los ensayos de vida útil. La acetona y el metanol fueron adquiridos de la marca TEDIA . El polímero Eudragit L 100-55 (Ácido metacrílico: Acrilato de etilo (1:1), PM 320,000 Da) fue amablemente donado por Evonik Industries. El hidróxido de sodio fue adquirido de la marca MERCK $^{\circ}$ y la fibra de SPME Carboxen/Polidimetilsiloxano (CAR/PDMS) de $75 \mu \mathrm{m}$ de la marca Supelco-Sigma Aldrich ${ }^{\circ}$

\section{Nanoformulación con eugenol y su incorporación en un recubrimiento comestible}

Las NP con EUG se prepararon mediante la técnica de nanoprecipitación (Fessi et al., 1989), para ello se inyectaron $4 \mathrm{~mL}$ de fase orgánica que contenía el polímero Eudragit $\mathrm{L}$ $100-55(55 \mathrm{mg})$ y el EUG $(60 \mathrm{mg})$ disueltos en acetona, en una fase acuosa $(25 \mathrm{~mL})$ que contenía el tensoactivo PVA al $0.5 \%$ $(p / p)$. La difusión de la fase orgánica en la fase acuosa indujo la agregación del polímero Eudragit L 100-55 y, por tanto, la encapsulación del EUG (NP-EUG) en su interior. Finalmente, el disolvente se eliminó utilizando un rotaevaporador (Control Laborota 4003, Heidolph Instruments, GER). Las NP sin EUG (NP-BCO) se obtuvieron siguiendo el mismo procedimiento descrito anteriormente.

El tamaño promedio de partícula y el índice de polidispersidad (IP) de las NP-EUG se midieron en un ángulo de dispersión de 90 grados utilizando la dispersión dinámica de la luz, mientras que, la medición del potencial zeta se realizó mediante microelectroforesis láser doppler (Zetasizer NanoZS90, Malvern Instruments, UK).

Para la formación del recubrimiento comestible se usó AL como agente formador. El AL sólido se incorporó en una dispersión acuosa de NP-EUG con agitación magnética hasta su completa disolución.

\section{Análisis de la nanoformulación por cromatografía de gases con detector de ionización de llama (CG-FID) y microextracción en fase sólida modalidad headspace (HS-SPME)}

Para determinar el porcentaje de eficiencia de encapsulación (\%EE), la dispersión de NP-EUG se centrifugó (Centrífuga Allegra 64R, Beckman Coulter, USA) y el sedimento formado por las NP-EUG se sometió a la técnica de HS-SPME utilizando una fibra de carboxen/polidimetilsiloxano (CAR/ PDMS) de $75 \mu \mathrm{m}$ de espesor de recubrimiento para cuantificar el EUG en las NP por CG-FID (Clarus 480, Perkin Elmer, USA). Para el método cromatográfico se utilizó una columna capilar (Elite-5, Perkin Elmer, USA) $(30 \mathrm{~m} \times 0.25 \mathrm{~mm} \times 0.25$ $\mu \mathrm{m})$. Las temperaturas del inyector $y$ del detector fueron de $270^{\circ} \mathrm{C}$. La temperatura del horno se programó de la siguiente manera: $70^{\circ} \mathrm{C}$ durante $1 \mathrm{~min}$, se incrementó en $30^{\circ} \mathrm{C} \mathrm{min}^{-1}$ a $190^{\circ} \mathrm{C}$, se incrementó en $10^{\circ} \mathrm{C} \mathrm{min}{ }^{-1}$ hasta $210^{\circ} \mathrm{C}$ y finalmente se aumentó en $20^{\circ} \mathrm{C} \mathrm{min}^{-1}$ hasta $270^{\circ} \mathrm{C}$ y se mantuvo durante $1 \mathrm{~min}$. El flujo del gas portador helio ( $99.999 \%$ de pureza, 
INFRA ${ }^{\circ}$ ) fue de $1 \mathrm{~mL} \mathrm{~min}{ }^{-1}$. Posteriormente, el porcentaje de eficiencia de encapsulación (\% EE) se calculó utilizando la siguiente fórmula:

$$
\% E E=(E U G C / E U G t) \times 100
$$

Donde EUGc es la cantidad EUG cuantificada en las NP-EUG (mg) y EUGt es la cantidad de EUG (mg) total utilizado en la fase orgánica de la nanoformulación.

Evaluación del efecto conservador de la nanoformulación en combinación con un recubrimiento comestible sobre los parámetros de vida útil del jitomate

Se seleccionaron jitomates (Solanum lycopersicum) con características homogéneas de color (maduración), tamaño y sin daño mecánico para la aplicación de los diferentes tratamientos. Se lavaron con agua destilada y se secaron. Los frutos se distribuyeron en cinco grupos de tres jitomates cada uno. El primer grupo se usó como CONTROL (sin tratamiento). El segundo grupo se puso en contacto por inmersión con una solución de AL (ALG) al $0.5 \%(p / p)$ por $1 \mathrm{~min}$. Al tercer grupo se le aplicó por inmersión, en las mismas condiciones anteriores, un tratamiento de RC de AL con EUG sin nanoencapsular (ALG-EUG). El cuarto grupo fue tratado con una dispersión de NP sin activo, es decir, sin EUG (NP-BCO). Finalmente, al quinto grupo se le aplicó por inmersión una dispersión acuosa del RC de AL al $0.5 \%(p / p)$ con la nanoformulación con EUG incorporado (ALG-NP-EUG). Todos los grupos se mantuvieron a $25^{\circ} \mathrm{C}$ y $35 \%$ de humedad durante 16 días. Transcurrido este tiempo, a cada grupo se le hicieron las evaluaciones mencionadas a continuación:

Evaluación de la firmeza. La firmeza de los jitomates se midió utilizando un Texturómetro (CT3 Texture Analyzer, Brookfield-Ametek, USA) equipado con una sonda cilíndrica de $2 \mathrm{~mm}$ de diámetro. La firmeza se expresó en Newton (N).

Evaluación del cambio de color. Los valores de color (CIE $L^{*} a^{*}$ y $b^{*}$ ) de los jitomates se determinaron por medición directa de la superficie del fruto utilizando un colorímetro (ColorFlex EZ, HunterLab, USA). El cambio de color total $(\Delta E)$ se midió utilizando la siguiente ecuación:

$$
\Delta \mathrm{E}^{*}=\left(\left(\mathrm{L}_{1}{ }_{1}-\mathrm{L}_{2}{ }_{2}\right)^{2}+\left(\mathrm{a}^{*}{ }_{1}-\mathrm{a}^{*}{ }_{2}\right)^{2}+\left(\mathrm{b}^{*}{ }_{1}-\mathrm{b}^{*}{ }_{2}\right)^{2}\right)^{1 / 2}
$$

Donde $\Delta \mathrm{E}^{*}$ es el cambio de color total. $\mathrm{L}^{*}{ }_{1} / \mathrm{L}^{*}$ es luminosidad inicial/luminosidad obtenida. $a^{*}{ }_{1} / a^{*}{ }_{2}$ es el color rojo-verde inicial/color rojo-verde obtenido y $b^{*}{ }_{1} / b^{*}{ }_{2}$ es el color amarillo-azul inicial/color amarillo-azul obtenido.

Evaluación del contenido de sólidos solubles totales. EI contenido de sólido soluble totales (SST) del jugo de jitomate se obtuvo de manera directa por refractometría (Abbemat 200, AntonPaar, AUT).

Evaluación de acidez titulable. Para el análisis de la calidad postcosecha de los frutos, se determinó la acidez titulable
(AT) mediante valoración del jugo de jitomate usando una solución de $\mathrm{NaOH}$ al $0.1 \mathrm{~N}$ hasta el final de la valoración $(\mathrm{pH}$ $=8.2$ ). El resultado se expresó en gramos de ácido cítrico por $100 \mathrm{~mL}$ de jugo.

Todos los parámetros se determinaron por triplicado al principio y al final del estudio de vida útil. Además, los resultados se analizaron en el programa estadístico Startical Product and Service Solutions (SPSS Statistics versión 23) mediante una ANOVA $(\rho=0.05)$.

Evaluación del efecto protector de la nanoformulación en combinación con el recubrimiento comestible sobre frutos de jitomate inoculados con el fitopatógeno Colletotrichum gloesporoides

Los frutos de jitomates fueron limpiados y desinfectados. Se les aplicó cada uno de los tratamientos por inmersión. En condiciones de asepsia, se realizaron tres heridas sobre su superficie. En una de ellas se inoculó el fitopatógeno Colletotrichum gloesporoides por estría y otra herida por punción. Los frutos se colocaron en una cámara de humedad a $25^{\circ} \mathrm{C}$ durante 5 días. Al finalizar, se observó ausencia o presencia del crecimiento del fitopatógeno en el jitomate.

\section{RESULTADOS Y DISCUSIÓN}

\section{Obtención y caracterización fisicoquímica de la nanofor- mulación con eugenol}

De manera general, los polímeros Eudragit han sido utilizados para modificar los perfiles de liberación de fármacos ofreciendo propiedades protectoras y de liberación sostenida (Piña-Barrera et al., 2019). Debido a las propiedades exhibidas por dichos polímeros (e.g. buena estabilidad, liberación controlada y enmascaramiento de sabor y olor), su uso confiere protección a los componentes bioactivos de naturaleza química inestable, no solo en la industria farmacéutica, sino también en la industria alimentaria. En el presente estudio se utilizó el polímero Eudragit L 100-55, el cual es un copolímero aniónico derivado del ácido acrílico y metacrílico (Figura 1).

Este polímero resulta ser muy atractivo para su uso en la industria alimentaria, ya que presenta excelentes pro-

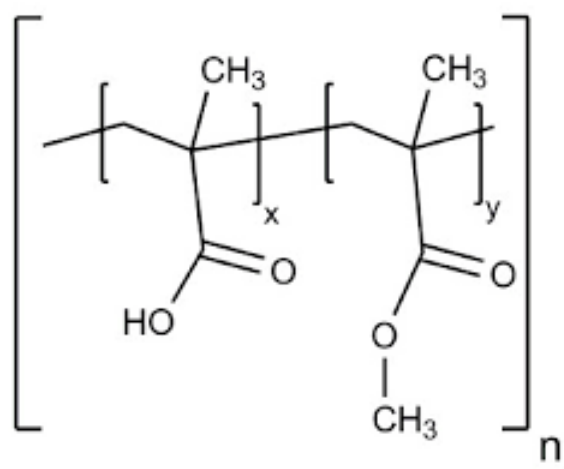

Figura 1. Estructura básica del polímero Eudragit L 10055 .

Figure 1. Basic structure of the polymer Eudragit L 10055. 
piedades de enmascaramiento de sabores desagradables, así como liberación controlada de forma dependiente del pH (Contri et al., 2013). En el presente estudio se obtuvieron NP-BCO con un tamaño promedio de $164.2 \pm 6.6 \mathrm{~nm}$, un IP de $0.084 \pm 0.018$ y un potencial zeta de $-1.80 \pm 0.64$. Las características fisicoquímicas para la nanoformulación NP-EUG se presentan en la Tabla 1.

Tabla 1. Características fisicoquímicas de las NP-BCO y de la nanoformulación con eugenol incorporado obtenida por la técnica de nanoprecipitación. $(n=3 ; \bar{x} \pm D S)$.

Table 1. Physicochemical characteristics of NP-BCO and nanoformulation with incorporated eugenol obtained by the nanoprecipitation technique. $(n=3 ; \bar{x} \pm D S)$

\begin{tabular}{ccccl}
\hline & $\begin{array}{c}\text { TAMAÑO } \\
(\mathbf{n m})\end{array}$ & IP1 & $\begin{array}{c}\text { POTENCIAL } \\
\text { ZETA } \\
(\mathbf{m V})\end{array}$ & $\%$ EE $^{2}$ \\
\hline NP-BCO & $164.0 \pm 6.6$ & $0.084 \pm 0.018$ & $-1.80 \pm 0.64$ & NA \\
NP-EUG & $171.0 \pm 3.0$ & $0.113 \pm 0.036$ & $-2.47 \pm 0.61$ & $31.85 \pm 12.77$ \\
\hline
\end{tabular}

' Índice de polidispersidad que varía de 0 a 1 . Un valor más alto corresponde a una distribución de tamaño menos homogénea; ${ }^{2}$ Porcentaje de eficiencia de encapsulación; NA: NP sin eugenol.

La técnica de nanoprecipitación ha sido utilizada exitosamente para la encapsulación de AE y sus componentes, como el EUG (Pascoli et al., 2018; Fraj et al., 2019; Lammari et al., 2020). La incorporación de estos componentes inestables al medio ambiente en NP puede ofrecer ventajas para su aplicación e incorporación en productos comerciales de la industria alimentaria. Por ejemplo, debido a su tamaño nanométrico y carácter multiparticulado, las NP cargadas con EUG pueden mejorar la interacción NP/superficie del fruto $y$, posteriormente liberar gradualmente el EUG (Boehm et al., 2003; Sotelo-Boyás et al., 2017). Además, en comparación con partículas grandes (e.g. micropartículas), los nanosistemas presentan una mejor relación superficie/volumen, por lo tanto, es posible tener mayor superficie del fruto en contacto directo con las NP (Granata et al., 2018). Además, la pared del polímero de las NP permite retener el EUG dentro de la estructura, disminuyendo así su velocidad de evaporación, favoreciendo su aplicación, aumentando el tiempo de residencia en la superficie del alimento y mejorando la incorporación del EUG en sistemas acuosos (He and Hwang, 2016). El tamaño promedio de las NP-EUG (i.e. $171.0 \pm 3.0 \mathrm{~nm}$ ) fue similar al reportado por Gomes, Moreira and Castell-Perez (2011) quienes obtuvieron NP de PLGA con EUG incorporado de un tamaño promedio de $179 \mathrm{~nm}$. Además, los autores reportan que en los ensayos de liberación realizados se presentó una tasa de liberación más lenta del EUG incorporado dentro de las NP, lo que mejoraría su aplicación general.

Por otro lado, el IP es un parámetro asociado a la homogeneidad de la dispersión del nanosistema. Para este estudio, el valor IP de las NP-EUG fue de $0.113 \pm 0.036$, lo que indica una alta homogeneidad de nanopartículas que permitiría que las interacciones individuales de las NP (e.g. bioadhesión y liberación de EUG) también sean homogéneas en la superficie del fruto. Para el equipo Zetasizer Nano-ZS90 (Malvern Instruments) utilizado, los valores de IP varían de 0 a 1. Un valor inferior a 0.200 indica una distribución homogénea del tamaño de nanopartículas (Galindo-Rodriguez et al., 2004).

El potencial electrostático en el límite que divide la capa compacta y la capa difusa de las partículas coloidales, llamado potencial zeta, fue de $-2.47 \mathrm{mV}$ para las NP-EUG. Este potencial negativo puede atribuirse a las moléculas del polímero aniónico formador de la pared polimérica (Eudragit L 100-55) que imparte una carga negativa a las NP obtenidas debido a sus grupos de ácido metacrílico. El potencial zeta de las NP depende principalmente de la naturaleza química del polímero, además de la naturaleza química del agente estabilizador. Por lo tanto, cuando se preparan NP a partir de polímeros derivados de metacrilatos utilizando agentes estabilizantes no iónicos, se obtienen valores de potencial zeta negativos debido a la presencia de grupos carboxílicos terminales del polímero (Mora-Huertas, Fessi and Elaissari, 2010) Por tanto, se deduce que el Eudragit formó efectivamente una envoltura que constituye la pared exterior de las nanopartículas. El núcleo corresponderá al EUG nanoencapsulado. De igual manera, el potencial zeta puede considerarse como un indicador de la estabilidad de las dispersiones de NP. Aunque se considera como regla general que valores absolutos alrededor de $30 \mathrm{mV}$ proporcionan una buena estabilidad, cuando se utilizan tensioactivos (i.e. PVA), que actúan principalmente por estabilización estérica, valores por debajo de $20 \mathrm{mV}$ o mucho más bajos pueden proporcionar una estabilización suficiente de las dispersiones (Galindo-Rodriguez et al., 2004; Honary and Zahir, 2013). Este potencial negativo también es importante porque podría facilitar la interacción del NP-EUG con la membrana de microorganismos fitopatógenos lo que aseguraría que la interacción del EUG fuera directamente desde la NP al microorganismo, mejorando, por lo tanto, su efectividad antimicrobiana (Wang, Hu and Shao, 2017).

Para completar la caracterización fisicoquímica de las NP-EUG se extrajo el EUG de las NP utilizando la técnica de HS-SPME y se cuantificó por un método analítico por CG-FID para determinar la eficiencia de encapsulación (\%EE) (Tabla 1) utilizado la ecuación (1). Con respecto a la técnica de HS-SPME, se utilizó una fibra CAR/PDMS de $75 \mu \mathrm{m}$ debido a que el EUG es un componente parcialmente polar (Log Po/w $=2.7)$ de bajo peso molecular $\left(164.20 \mathrm{~g} \mathrm{~mol}^{-1}\right)$ que tiene afinidad por este tipo de fibras. En la Figura 2 se muestra el cromatograma del EUG extraído por la técnica de HS-SPME

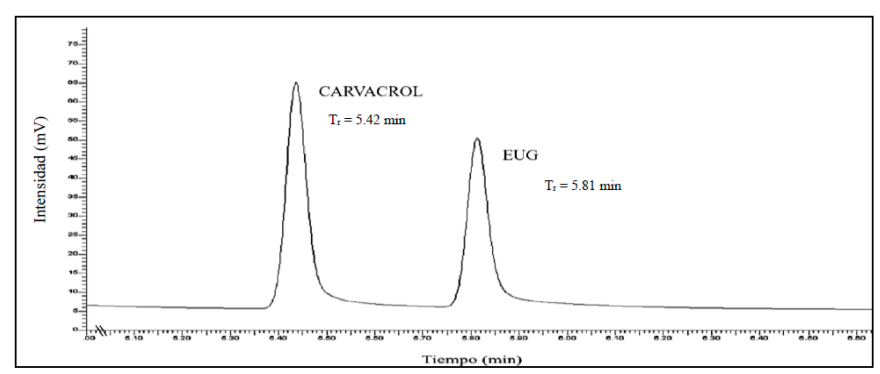

Figura 2. Cromatograma del EUG $\left(40 \mu \mathrm{g} \mathrm{mL}^{-1}\right)$ y carvacrol $\left(10 \mu \mathrm{g} \mathrm{mL}^{-1}\right)$ con el recubrimiento de CAR/PDMS $(75 \mu \mathrm{m})$ por CG-FID.

Figure 2. Chromatogram of EUG $\left(40 \mu \mathrm{g} \mathrm{mL}^{-1}\right)$ and carvacrol $\left(10 \mu \mathrm{g} \mathrm{mL}^{-1}\right)$ coated with CAR/PDMS $(75 \mu \mathrm{m})$ by GC-FID. 
y el estándar interno (carvacrol) utilizado para determinar el \%EE por el método analítico por CG-FID (Figura 2).

El \%EE obtenido para las NP-EUG fue de $31.85 \pm 12.77$ (Tabla 1) lo que indica que más del $30 \%$ del EUG añadido durante la formulación quedó encapsulado en la NP utilizando la técnica de nanoprecipitación, por lo tanto, es probable que este compuesto activo se libere gradualmente desde la NP a la superficie de la fruta.

Evaluación del efecto conservador de la nanoformulación en combinación con un recubrimiento comestible sobre los parámetros de vida útil del jitomate (Solanum lycopersicum)

Firmeza. En la Figura 3 se detallan los resultados de los ensayos de firmeza realizados en jitomates después de 16 días de almacenamiento a temperatura ambiente. La firmeza de los jitomates fue de $1.5 \mathrm{~N}$ al comienzo del experimento. Este parámetro disminuyó para todos los grupos de jitomates analizados. Se observó una disminución del $42.7 \%$ de la firmeza en los jitomates sin ningún tratamiento (CTRL) $(0.86$ $\mathrm{N})$. Los jitomates tratados con ALG $(0.68 \mathrm{~N})$ y con ALG-EUG $(0.71 \mathrm{~N})$ presentaron una pérdida del 54.7 y $52.7 \%$ respectivamente, mientras que los jitomates tratados con NP-BCO $(0.87 \mathrm{~N})$ tuvieron un valor de firmeza similar a la inicial. No se encontró diferencia significativa entre los grupos ALG, ALGEUG y NP-BCO respecto al grupo CTRL.

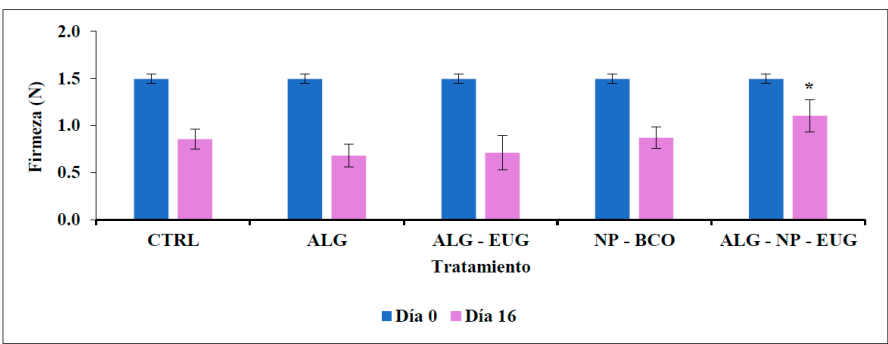

Figura 3. Firmeza, día 0 y día 16 , de jitomates después del almacenamiento durante 16 días a $25^{\circ} \mathrm{C}$. CTRL = sin tratamiento, $A L G=$ alginato, ALG-EUG = alginato + eugenol libre, NP-BCO $=\mathrm{NP}$ sin activo, $\mathrm{ALG}-\mathrm{NP}-\mathrm{EUG}=$ alginato $+\mathrm{NP}$ con eugenol. $(n=3 ; \bar{x} \pm D S)$.

* Diferencia significativa respecto al grupo CTRL.

Figure 3. Firmness, day 0 and day 16, of tomatoes after storage for 16 days at $25^{\circ} \mathrm{C} . \mathrm{CTRL}=$ no treatment, $\mathrm{ALG}=$ alginate, $\mathrm{ALG}-\mathrm{EUG}=$ alginate + free eugenol, NP-BCO $=$ NP without active, ALG-NP-EUG = alginate $+N P$ with eugenol. $(n=3 ; \bar{x} \pm D S)$.

*Significant difference with respect to the CTRL group.

Los jitomates tratados con las NP-EUG en combinación con el RC (ALG-NP-EUG) presentaron una mayor firmeza $(1.10 \mathrm{~N})$ en comparación a los cuatro tratamientos anteriores, representando tan solo un $22 \%$ de pérdida en comparación con los frutos sin tratamiento (CTRL). Esta diferencia fue estadísticamente significativa. La firmeza de las frutas y hortalizas está relacionada con la estructura de la pared celular, la cual depende de la turgencia, la cohesión, la forma y el tamaño de las células que la conforman. La pérdida de agua está relacionada íntimamente con la pérdida de turgencia de las células del mesocarpio, disminuyendo la firmeza del fruto (Vicente et al., 2007). En este trabajo, la presencia del RC ALG-NP-EUG pudo interferir con la disminución en la taza de transpiración del fruto, lo que resultó en una menor pérdida de firmeza por parte de los jitomates tratados. De igual manera, la pérdida de firmeza está relacionada a un aumento de la actividad de las enzimas hidrolíticas (i.e. poligalacturonasa) que actúan sobre las pectinas de la pared celular, lo que ocasiona cambios en los tejidos, que a su vez provoca el ablandamiento del fruto (Navarro-López et al., 2012). Esta actividad enzimática es baja durante la primera etapa del desarrollo del fruto, luego aumenta y alcanza un máximo en la etapa climatérica del proceso de maduración (Yao et al., 2014). Resultados similares fueron reportados Fagundes et al., (2014) quienes obtuvieron resultados de firmeza similares a los iniciales en tomates tratados con recubrimientos de hidroxipropil metilcelulosa y cera de abejas después de 15 días de almacenamiento. Los autores atribuyen esta retención de firmeza en los tomates recubiertos a la reducción en las actividades enzimáticas causada por la modificación de la atmósfera interna de la fruta. Es decir, a una menor tasa de respiración. De igual manera, en nuestro estudio, el AL del RC ALG-NP-EUG tuvo la capacidad de actuar como una barrera que interfirió el intercambio gaseoso, lo cual condujo a la reducción de la tasa de respiración de los jitomates y evitó la pérdida de agua; además, es posible que la actividad biológica del EUG nanoencapsulado disminuyera la actividad enzimática del fruto, dando como resultados una maduración más lenta.

Cambio de color. En cuanto al color, en la industria alimentaria, para medir el cambio de color en un producto como el jitomate, se han establecido coordenadas expresadas en valores numéricos que permiten correlacionar el color con la madurez. En este estudio, los cambios de color fueron determinados por la escala de color CIE $L a^{*} b^{*}$, donde $a^{*}$ es la coordenada roja/verde ( $+a^{*}$ indica más rojo y $-a^{*}$ indica más verde), mientras que $b^{*}$ es la coordenada amarilla/azul (+b* indica más amarillo y $-b^{*}$ indica más azul). En la Figura 4 se muestra el análisis de los 3 valores de las coordenadas obtenidas, presentados como el cambio de color $(\Delta \mathrm{E})$, utilizando la ecuación (2), producido en cada uno de los

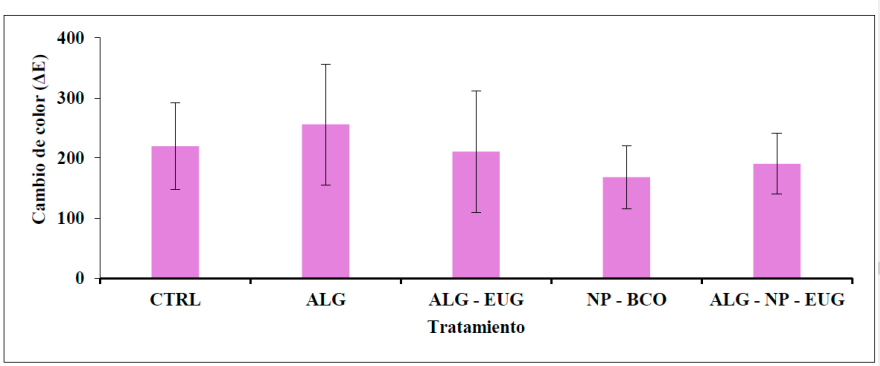

Figura 4. Cambio de color de jitomates después del almacenamiento durante 16 días a $25^{\circ} \mathrm{C}$. CTRL = sin tratamiento, $A L G=$ alginato, $A L G-E U G=$ alginato + eugenol libre, NP-BCO $=$ NP sin activo, ALG-NP-EUG $=$ alginato $+\mathrm{NP}$ con eugenol. $(n=3 ; \bar{x} \pm D S)$.

Figure 4. Color change of tomatoes after storage for 16 days at $25^{\circ} \mathrm{C}$. CTRL $=$ no treatment, $A L G=$ alginate, $A L G-E U G=$ alginate + free eugenol, NP$\mathrm{BCO}=\mathrm{NP}$ without active, ALG-NP-EUG $=$ alginate $+\mathrm{NP}$ with eugenol. $(n=3$; $\bar{X} \pm D S)$. 
grupos tratados y almacenados por 16 días. Se obtuvo un valor $\Delta \mathrm{E}$ de 219.75 para el CTRL, 255.71 para ALG y 210.80 para ALG-EUG, 167.76 para el NP-BCO y 190.47 para el RC ALG-NP-EUG. Un menor cambio de color es interpretado como una preservación de la calidad del jitomate. Aunque no hubo una diferencia significativa entre los grupos, puede observarse que la presencia de las NP en combinación con el $\mathrm{RC}$ de $A L G$ en la superficie del fruto pudieron tener un efecto en la conservación del color. Es importante mencionar que el jitomate, al alcanzar su madurez comercial, sufre cambios mínimos en cuanto a su coloración, algo característico en los frutos climatéricos. Diferentes autores han mencionado que la aplicación de recubrimientos puede retrasar los cambios de color en los tomates durante el almacenamiento al crear una atmósfera modificada en la fruta (Ali et al., 2010; Gomes, Moreira and Castell-Perez, 2011)

Sólidos solubles totales y acidez titulable. Los SST de las frutas tienden a aumentar durante la maduración. El valor de SST (Figura 5) para los jitomates al comienzo del experimento fue de $3.64{ }^{\circ}$ Brix. Después de 16 días de almacenamiento, este parámetro aumentó para los jitomates control (4.83 'Brix), así como para los jitomates tratados con el ALG-EUG (4.74 $\left.{ }^{\circ} \mathrm{Brix}\right)$, ALG $\left(5.21^{\circ} \mathrm{Brix}\right), \mathrm{NP}-\mathrm{CO}\left(5.08^{\circ} \mathrm{Brix}\right)$ y el RC ALGNP-EUG ( $4.31^{\circ}$ Brix).

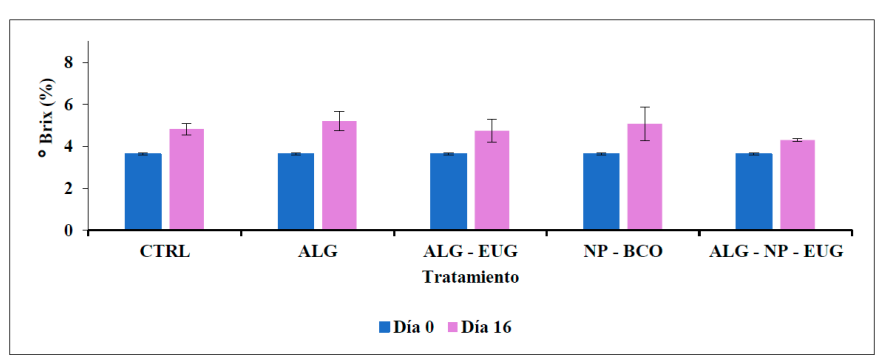

Figura 5. Solidos solubles totales, día 0 y día 16 , de jitomates después del almacenamiento durante 16 días a $25^{\circ} \mathrm{C}$. CTRL = sin tratamiento, $\mathrm{ALG}=$ alginato, $\mathrm{ALG}-\mathrm{EUG}=$ alginato + eugenol libre, NP-BCO $=\mathrm{NP}$ sin activo, ALG-NP-EUG = alginato + NP con eugenol. $(n=3 ; \bar{x} \pm D S)$.

Figure 5. Total soluble solids, day 0 and day 16 , of tomatoes after storage for 16 days at $25^{\circ} \mathrm{C}$. CTRL $=$ no treatment, $A L G=$ alginate, $A L G-E U G=$ alginate + free eugenol, NP-BCO $=$ NP without active, ALG-NP-EUG $=$ alginate $+\mathrm{NP}$ with eugenol.

$(n=3 ; \bar{x} \pm D S)$.

El aumento de los SST durante el almacenamiento es debido a la respiración. Durante el proceso de maduración, la acumulación de azúcar dependerá de la degradación del almidón, principal compuesto de almacenamiento energético en el jitomate verde. Además, la actividad metabólica continúa como resultado de la maduración de la fruta, lo que conduce a la conversión de carbohidratos y ácidos orgánicos en azúcares para ser utilizados en varios procesos metabólicos (Guerreiro et al., 2015). Por otro lado, comparado con el grupo control, el recubrimiento en combinación con las NP-EUG (ALG-NP-EUG), mostró los valores más bajos de SST. Este comportamiento podría atribuirse a la presencia del RC de AL y una sinergia con las NP-EUG, lo que ocasiona una desaceleración más efectiva de la respiración y la actividad metabólica, retrasando el proceso de maduración de los frutos. Estos resultados son similares a lo reportado por Sucharitha, Beulah and Ravikiran (2018) quienes trataron tomates con recubrimientos de quitosano y reportaron una diferencia significativa al final del período de almacenamiento (15 días) entre las muestras revestidas y no revestidas con el quitosano. En nuestro estudio, se observó una diferencia de los SST entre el RC ALG-NP-EUG (4.31 ${ }^{\circ}$ Brix) y el ALG solo (5.21 ${ }^{\circ}$ Brix). Esto podría deberse a que la presencia de las NP con EUG encapsulado en la superficie del fruto se prolongue por más tiempo y que, además de las propiedades de barrera del recubrimiento, la liberación sostenida del EUG de las NP podría haber extendido la actividad biológica de EUG (e.g. antioxidante) en la superficie del jitomate, lo que contribuyó a tener una mejor conservación de los frutos.

Finalmente, como se muestra en la Figura 6, la AT de

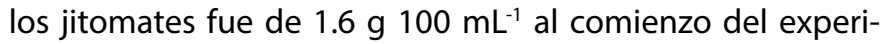
mento y después de 16 días de almacenamiento, los valores de AT disminuyeron para todos los grupos. Esta reducción de los valores de AT está asociada al metabolismo de los ácidos orgánicos en las frutas durante el proceso de maduración. Lo ácidos orgánicos son responsables de la acidez de la fruta que se expresa como AT. Durante la maduración de los jitomates, la cantidad de ácidos orgánicos disminuye, y esto es debido a que los ácidos orgánicos son metabolizados, principalmente, para asegurar el suministro adicional de carbono para la obtención de sacarosa, glucosa y fructosa en la fruta (Fabi et al., 2007). En cuanto a los grupos de jitomates con tratamientos, los resultados de AT tuvieron la misma tendencia que los obtenidos para SST. Después de 16 días de almacenamiento, los jitomates del grupo control tuvieron la disminución más importante de AT, mientras que las del ALG-NP-EUG la disminución más baja de AT. Esto significa que el recubrimiento en combinación con las NP-EUG fue el tratamiento más efectivo para retrasar la maduración, lo que puede atribuirse a la presencia del recubrimiento de AL que actuó como una barrera y disminuyó la tasa de respiración (metabolismo) de los jitomates.

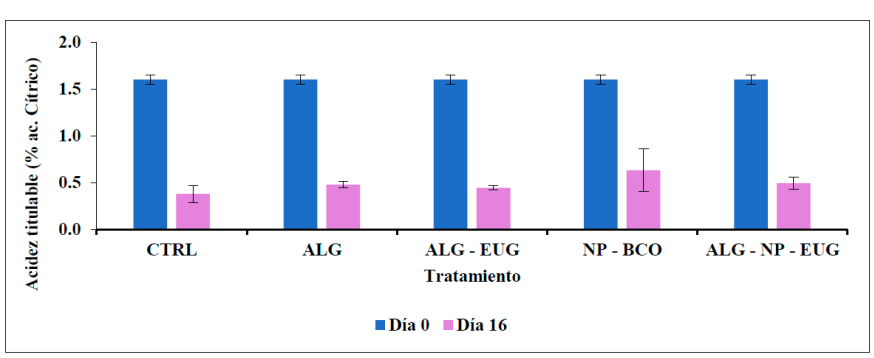

Figura 6. Acidez titulable, día 0 y día 16 , de jitomates después del almacenamiento durante 16 días a $25^{\circ} \mathrm{C}$. CTRL $=$ sin tratamiento, $\mathrm{ALG}=$ alginato, $\mathrm{ALG}-\mathrm{EUG}=$ alginato + eugenol libre, NP-BCO $=\mathrm{NP} \sin$ activo, $\mathrm{ALG}-\mathrm{NP}-\mathrm{EUG}$ $=$ alginato + NP con eugenol.

$(n=3 ; \bar{x} \pm D S)$.

Figure 6. Titratable acidity, day 0 and day 16 , of tomatoes after storage for 16 days at $25^{\circ} \mathrm{C} . \mathrm{CTRL}=$ no treatment, $\mathrm{ALG}=$ alginate, $\mathrm{ALG}-\mathrm{EUG}=$ alginate + free eugenol, NP-BCO $=$ NP without active, ALG-NP-EUG $=$ alginate + NP with eugenol. $(n=3 ; \bar{x} \pm D S)$. 
Evaluación del efecto protector de la nanoformulación en combinación con el recubrimiento comestible sobre frutos de jitomate inoculados con el fitopatógeno Colletotrichum gloesporoides.

El cultivo de jitomate es afectado por diversas enfermedades postcosecha, las cuales muchas de ellas son ocasionadas por hongos. Entre los principales agentes fitopatógenos postcosecha del jitomate se encuentran: Fusarium solani, Botritys cinérea, Alternaria alternata, Penicilium expansum y, de manera particular, Colletotrichum gloesporoides (Barad et al., 2017). En el presente estudio, jitomates con cada uno de los tratamientos antes mencionados se les inoculó por punción el fitopatógeno Colletotrichum gloesporoides y se observó su desarrollo durante 5 días a temperatura ambiente en cámaras de humedad. Los resultados se observan en la Figura 7. Después de 5 días, los jitomates tratados con el RC ALG-NP-EUG no presentaron crecimiento del hongo (Figura 7-E), mientras que el resto de los frutos presentaron colonias características del hongo Colletotrichum gloeosporioides. Esta protección se debe a la capacidad antimicrobiana y el efecto sobre fitopatógenos del EUG (Abbaszadeh et al., 2014; Marchese et al., 2017; Davy et al., 2020). Hong et al., 2015 demostraron que el $\mathrm{AE}$ de clavo y el eugenol presentaron una importante inhibición del crecimiento de Colletotrichum gloeosporioides por medio de la reducción del diámetro de una lesión en pimiento verde inmaduros inoculados con este fitopatógeno. Además, la incorporación del EUG en la estructura polimérica de la NP puede ocasionar un aumento en el tiempo de residencia en la superficie del fruto al disminuir su rápida evaporación al aplicarse de manera libre.

Se ha propuesto que el mecanismo de acción de la actividad antifúngica de los componentes de los $A E$ parece depender de su estructura química y de su capacidad para atravesar la pared celular y penetrar entre las cadenas de ácidos grasos de la bicapa lipídica, haciendo que la membrana celular sea mucho más permeable y, como resultado, ocasio-

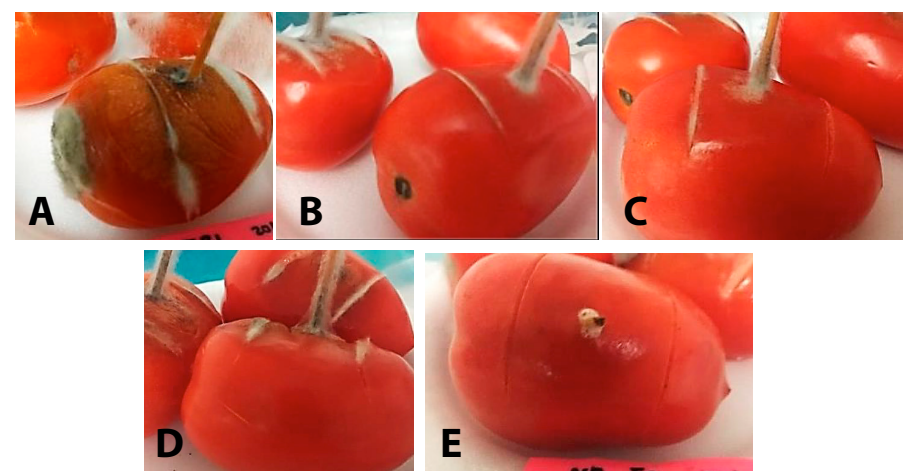

Figura 7. Prueba de patogenicidad con C. gloesporoides después de 5 días de almacenamiento de tomates con diferentes tratamientos. $A$ ) = control, $B)=$ alginato, C) = alginato + eugenol libre, D) = NP blanco, E) ALG-NP-EUG. $(n=3 ; \bar{x} \pm D S$ ).

Figure 7. Pathogenicity test with $C$. gloesporoides after 5 days of storage of tomatoes with different treatments. $\mathrm{A})=$ control, $\mathrm{B})=$ alginate, $\mathrm{C}$ ) = alginate + free eugenol, $\mathrm{D})=$ blank NP, E) ALG-NP-EUG. $(n=3 ; \bar{x} \pm D S)$. nan la muerte celular o la inhibición de la esporulación y la germinación de hongos u otros microorganismos causantes del deterioro (Wattanasatcha, Rengpipat and Wanichwecharungruang, 2012; Diao et al., 2014; Grande-Tovar et al., 2018). Tomando en cuenta este mecanismo, el EUG incorporado en las NP en combinación con el RC ayudó a tener un contacto más íntimo y prolongado entre el activo y los microorganismos en la superficie de la fruta.

\section{CONCLUSIONES}

Las propiedades fisicoquímicas de los componentes individuales del RC en combinación con NP-EUG contribuyeron positivamente a retrasar la maduración de los jitomates y protegerlos de fitopatógenos como el Colletotrichum gloesporoides. Mientras que el RC de AL actuó como una barrera que redujo la transpiración y el metabolismo de las frutas, el EUG con actividad antimicrobiana evitó el crecimiento del microorganismo fitopatógeno. Además de esto, debido a su tamaño y carácter multiparticulado, las NP con EUG incorporado pudieron distribuirse de manera más uniforme en la superficie del fruto, liberando al EUG de manera gradual y aumentando su tiempo de residencia en la fruta. Esto demuestra que el EUG nanoencapsulado en combinación con $\mathrm{RC}$ son una alternativa favorable a los métodos tradicionales de conservación.

\section{AGRADECIMIENTOS}

Al financiamiento otorgado mediante los proyectos UANL-PAICYT IT1919-21 y CN1614-21 y al CONACYT por la beca 273928.

\section{REFERENCIAS}

Abbaszadeh, S. et al. (2014) 'Antifungal efficacy of thymol, carvacrol, eugenol and menthol as alternative agents to control the growth of food-relevant fungi', Journal de Mycologie Medicale. Elsevier Masson SAS, 24(2), pp. e51-e56. doi: 10.1016/j.mycmed.2014.01.063.

Ali, A. et al. (2010) 'Gum arabic as a novel edible coating for enhancing shelf-life and improving postharvest quality of tomato (Solanum lycopersicum L.) fruit', Postharvest Biology and Technology. Elsevier, 58(1), pp. 42-47. doi: 10.1016/j. postharvbio.2010.05.005.

Asbahani, A. El et al. (2015) 'Essential oils: From extraction to encapsulation', International Journal of Pharmaceutics. Elsevier B.V., 483(1-2), pp. 220-243. doi: 10.1016/j. ijpharm.2014.12.069.

Barad, S. et al. (2017) ‘Differential gene expression in tomato fruit and Colletotrichum gloeosporioides during colonization of the RNAi-SIPH tomato line with reduced fruit acidity and higher $\mathrm{pH}^{\prime}, B M C$ Genomics. BioMed Central Ltd., 18(1). doi: 10.1186/s12864-017-3961-6.

Boehm, A. L. et al. (2003) 'Nanoprecipitation technique for the encapsulation of agrochemical active ingredients', Journal of Microencapsulation, 20(4), pp. 433-441. doi: $10.1080 / 0265204021000058410$. 
Camele, I. et al. (2012) 'In vitro control of post-harvest fruit rot fungi by some plant essential oil components', International Journal of Molecular Sciences. Molecular Diversity Preservation International, 13(2), pp. 2290-2300. doi: 10.3390/ijms13022290.

Choudhary, D. K. et al. (2017) Volatiles and food security: Role of volatiles in agro-ecosystems, Volatiles and Food Security: Role of Volatiles in Agro-Ecosystems. Springer Singapore. doi: 10.1007/978-981-10-5553-9.

Contri, R. V. et al. (2013) 'Vegetable oils as core of cationic polymeric nanocapsules: Influence on the physicochemical properties', Journal of Experimental Nanoscience, 8(7-8), pp. 913-924. doi: 10.1080/17458080.2011.620019.

Correa-Pacheco, Z. N. et al. (2017) 'The Effect of Nanostructured Chitosan and Chitosan-thyme Essential Oil Coatings on Colletotrichum gloeosporioides Growth in vitro and on cv Hass Avocado and Fruit Quality', Journal of Phytopathology. Blackwell Publishing Ltd, 165(5), pp. 297-305. doi: 10.1111/ jph.12562.

Davy, M. et al. (2020) 'Antifungal effects of clove (Syzygium aromaticum) essential oil against Colletotrichum gloeosporioides, the fungus associated with papaya (Carica papaya L.) fruit anthracnose', International Journal of Applied Microbiology and Biotechnology Research, pp. 51-57. doi: 10.33500/ijambr.2020.08.005.

Diao, W. R. et al. (2014) 'Chemical composition, antibacterial activity and mechanism of action of essential oil from seeds of fennel (Foeniculum vulgare Mill.)', Food Control. Elsevier Ltd, 35(1), pp. 109-116. doi: 10.1016/j.foodcont.2013.06.056.

Fabi, J. P. et al. (2007) 'Papaya fruit ripening: Response to ethylene and 1-methylcyclopropene (1-MCP)', Journal of Agricultural and Food Chemistry, 55(15), pp. 6118-6123. doi: 10.1021/ jf070903c.

Fagundes, C. et al. (2014) 'Effect of antifungal hydroxypropyl methylcellulose-beeswax edible coatings on gray mold development and quality attributes of cold-stored cherry tomato fruit', Postharvest Biology and Technology. Elsevier, 92, pp. 1-8. doi: 10.1016/j.postharvbio.2014.01.006.

FAO (2011) Global food losses and food waste - Extent, causes and prevention, Food and Agriculture Organization of the United Nations. Roma. doi: 10.1098/rstb.2010.0126.

Fessi, H. et al. (1989) 'Nanocapsule formation by interfacial polymer deposition following solvent displacement', International Journal of Pharmaceutics. Elsevier, 55(1), pp. R1-R4. doi: 10.1016/0378-5173(89)90281-0.

Feyzioglu, G. C. and Tornuk, F. (2016) ‘Development of chitosan nanoparticles loaded with summer savory (Satureja hortensis L.) essential oil for antimicrobial and antioxidant delivery applications', LWT - Food Science and Technology. Academic Press, 70, pp. 104-110. doi: 10.1016/j.lwt.2016.02.037.

Fraj, A. et al. (2019) 'A comparative study of oregano (Origanum vulgare L.) essential oil-based polycaprolactone nanocapsules/ microspheres: Preparation, physicochemical characterization, and storage stability', Industrial Crops and Products. Elsevier B.V., 140, p. 111669. doi: 10.1016/j. indcrop.2019.111669.

Galindo-Rodriguez, S. et al. (2004) 'Physicochemical parameters associated with nanoparticle formation in the salting-out, emulsification-diffusion, and nanoprecipitation methods', Pharmaceutical Research, 21(8), pp. 1428-1439. doi: 10.1023/B:PHAM.0000036917.75634.be.
Gomes, C., Moreira, R. G. and Castell-Perez, E. (2011) ‘Poly (DLlactide-co-glycolide) (PLGA) Nanoparticles with Entrapped trans-Cinnamaldehyde and Eugenol for Antimicrobial Delivery Applications', Journal of Food Science. John Wiley \& Sons, Ltd, 76(2), pp. N16-N24. doi: 10.1111/j.17503841.2010.01985.x.

Granata, G. et al. (2018) 'Essential oils encapsulated in polymerbased nanocapsules as potential candidates for application in food preservation', Food Chemistry. Elsevier, 269, pp. 286292. doi: https://doi.org/10.1016/j.foodchem.2018.06.140.

Grande-Tovar, C. D. et al. (2018) 'Chitosan coatings enriched with essential oils: Effects on fungi involve in fruit decay and mechanisms of action', Trends in Food Science and Technology. Elsevier, 78(May), pp. 61-71. doi: 10.1016/j.tifs.2018.05.019.

Guerreiro, A. C. et al. (2015) 'The effect of alginate-based edible coatings enriched with essential oils constituents on Arbutus unedo L. fresh fruit storage', Postharvest Biology and Technology. Elsevier, 100, pp. 226-233. doi: 10.1016/j. postharvbio.2014.09.002.

He, X. and Hwang, H. M. (2016) 'Nanotechnology in food science: Functionality, applicability, and safety assessment', Journal of Food and Drug Analysis. Elsevier, 24(4), pp. 671-681. doi: 10.1016/j.jfda.2016.06.001.

Honary, S. and Zahir, F. (2013) 'Effect of Zeta Potential on the Properties of Nano-Drug Delivery Systems - A Review (Part 2)', Tropical Journal of Pharmaceutical Research, 12(April), pp. 255-264. doi: 10.4314/tjpr.v12i2.20.

Hong, J. K. et al. (2015) 'Application of volatile antifungal plant essential oils for controlling pepper fruit anthracnose by Colletotrichum gloeosporioides', Plant Pathology Journal. Korean Society of Plant Pathology, 31(3), pp. 269-277. doi: 10.5423/PPJ.OA.03.2015.0027.

Ju, J. et al. (2019) 'Application of edible coating with essential oil in food preservation', Critical Reviews in Food Science and Nutrition. Taylor \& Francis, 59(15), pp. 2467-2480. doi: 10.1080/10408398.2018.1456402.

Lammari, N. et al. (2020) 'Encapsulation of essential oils via nanoprecipitation process: Overview, progress, challenges and prospects', Pharmaceutics. MDPI AG, p. 431. doi: 10.3390/ pharmaceutics12050431.

Marchese, A. et al. (2017) 'Antimicrobial activity of eugenol and essential oils containing eugenol: A mechanistic viewpoint', Critical Reviews in Microbiology. Taylor and Francis Ltd, pp. 668-689. doi: 10.1080/1040841X.2017.1295225.

Mora-Huertas, C. E., Fessi, H. and Elaissari, A. (2010) 'Polymerbased nanocapsules for drug delivery', International Journal of Pharmaceutics, 385(1-2), pp. 113-142. doi: 10.1016/j. ijpharm.2009.10.018.

Navarro-López, E. R. et al. (2012) ‘Calidad poscosecha en frutos de tomate hidropónico producidos con agua residual y de pozo', Revista Chapingo, Serie Horticultura. Universidad Autónoma Chapingo, 18(3), pp. 263-277. doi: 10.5154/r. rchsh.2009.11.097.

Pandey, A. K. et al. (2017) 'Essential oils: Sources of antimicrobials and food preservatives', Frontiers in Microbiology. Frontiers, 7(JAN), p. 2161. doi: 10.3389/fmicb.2016.02161.

Pascoli, M. et al. (2018)'State of the art of polymeric nanoparticles as carrier systems with agricultural applications: a minireview', Energy, Ecology and Environment. Joint Center on Global Change and Earth System Science of the University of Maryland and Beijing Normal University, 3(3), pp. 137-148. doi: 10.1007/s40974-018-0090-2. 
Piña-Barrera, A. M. et al. (2019) 'Application of a multisystem coating based on polymeric nanocapsules containing essential oil of Thymus vulgaris L. to increase the shelf life of table grapes (Vitis vinifera L.)', IEEE Transactions on NanoBioscience. Institute of Electrical and Electronics Engineers (IEEE), 18(4), pp. 549-557. doi: 10.1109/ tnb.2019.2941931.

Prates, L. H. F. et al. (2019) 'Eugenol diffusion coefficient and its potential to control Sitophilus zeamais in rice', Scientific reports. NLM (Medline), 9(1), p. 11161. doi: 10.1038/s41598019-47562-1.

Sotelo-Boyás, M. E. et al. (2017)’Physicochemical characterization of chitosan nanoparticles and nanocapsules incorporated with lime essential oil and their antibacterial activity against food-borne pathogens, LWT - Food Science and Technology. Academic Press, 77, pp. 15-20. doi: 10.1016/j. Iwt.2016.11.022.

Sucharitha, K. V., Beulah, A. M. and Ravikiran, K. (2018) 'Effect of chitosan coating on storage stability of tomatoes (Lycopersicon esculentum Mill)', International Food Research Journal, 25(1), pp. 93-99.
Vicente, A. R. et al. (2007) 'The linkage between cell wall metabolism and fruit softening: Looking to the future', Journal of the Science of Food and Agriculture. John Wiley \& Sons, Ltd, 87(8), pp. 1435-1448. doi: 10.1002/jsfa.2837.

Wang, L., Hu, C. and Shao, L. (2017) 'The antimicrobial activity of nanoparticles: Present situation and prospects for the future', International Journal of Nanomedicine. Dove Press, 12, pp. 1227-1249. doi: 10.2147/IJN.S121956.

Wattanasatcha, A., Rengpipat, S. and Wanichwecharungruang, S. (2012) 'Thymol nanospheres as an effective anti-bacterial agent', International Journal of Pharmaceutics. Elsevier B.V., 434(1-2), pp. 360-365. doi: 10.1016/j.jjpharm.2012.06.017.

Yao, B. N. et al. (2014) 'The role of hydrolases in the loss of firmness and of the changes in sugar content during the post-harvest maturation of Carica papaya L. var solo 8', Journal of Food Science and Technology. Springer, 51(11), pp. 3309-3316. doi: 10.1007/s13197-012-0858-x.

Zia, K. M. et al. (2015) 'Alginate based polyurethanes: A review of recent advances and perspective', International Journal of Biological Macromolecules. Elsevier B.V., pp. 377-387. doi: 10.1016/j.ijbiomac.2015.04.076. 